\title{
Amyloid-Independent Mechanisms in Alzheimer's Disease Pathogenesis
}

\author{
Sanjay W. Pimplikar, ${ }^{1}$ Ralph A. Nixon, ${ }^{2}$ Nikolaos K. Robakis, ${ }^{3}$ Jie Shen, ${ }^{4}$ and Li-Huei Tsai ${ }^{5}$ \\ ${ }^{1}$ Department of Neuroscience, Lerner Research Institute, Cleveland Clinic, and Cleveland Clinic Lerner College of Medicine of Case Western Reserve \\ University, Cleveland, Ohio 44195, ${ }^{2}$ Center for Dementia Research, Nathan S. Kline Institute, Orangeburg, New York, 10962, and Departments of Psychiatry \\ and Cell Biology, New York University Langone Medical Center, New York, New York 10016, ${ }^{3}$ Departments of Psychiatry and Neuroscience, Center for \\ Molecular Biology and Genetics of Neurodegeneration, Mount Sinai School of Medicine, New York, New York 10029, ${ }^{4}$ Center for Neurologic Diseases, \\ Program in Neuroscience, Brigham and Women's Hospital, Harvard Medical School, Boston, Massachusetts 02115, and 5Department of Brain and \\ Cognitive Sciences, Picower Institute for Learning and Memory, Massachusetts Institute of Technology, and Howard Hughes Medical Institute, Cambridge, \\ Massachusetts 02139
}

Despite the progress of the past two decades, the cause of Alzheimer's disease (AD) and effective treatments against it remain elusive. The hypothesis that amyloid- $\beta(\mathrm{A} \beta)$ peptides are the primary causative agents of AD retains significant support among researchers. Nonetheless, a growing body of evidence shows that $\mathrm{A} \beta$ peptides are unlikely to be the sole factor in $\mathrm{AD}$ etiology. Evidence that $\mathrm{A} \beta /$ amyloidindependent factors, including the actions of $\mathrm{AD}$-related genes, also contribute significantly to $\mathrm{AD}$ pathogenesis was presented in a symposium at the 2010 Annual Meeting of the Society for Neuroscience. Here we summarize the studies showing how amyloidindependent mechanisms cause defective endo-lysosomal trafficking, altered intracellular signaling cascades, or impaired neurotransmitter release and contribute to synaptic dysfunction and/or neurodegeneration, leading to dementia in AD. A view of AD pathogenesis that encompasses both the amyloid-dependent and -independent mechanisms will help fill the gaps in our knowledge and reconcile the findings that cannot be explained solely by the amyloid hypothesis.

Alzheimer's disease (AD) is a progressive neurodegenerative disorder that begins as mild short-term memory deficits and culminates in total loss of cognition and executive functions. Currently, the precise cause of the disease is not known and there is no cure. Genetic studies (Price et al., 1998) have identified mutations in amyloid precursor protein (APP) and presenilin 1 and 2 (PS1, PS2) that cause rare, dominantly inherited familial AD (FAD). Proteolytic processing of APP by BACE ( $\beta$-site APP cleaving enzyme) followed by PS-containing $\gamma$-secretase complex generates amyloid- $\beta$ (A $\beta$ ) peptides that deposit in amyloid plaques. Genetic and cell biological studies show increased production of more amyloidogenic $\mathrm{A} \beta$ peptides associated with FAD-linked mutations, providing strong support for the amyloid hypothesis (Hardy and Selkoe, 2002), which posits that A $\beta$ peptides play a pivotal role in $\mathrm{AD}$ pathogenesis. However, $\mathrm{A} \beta$ peptides are also generated as a part of normal metabolism and there is no consensus regarding the identity of the disease-causing, pathological form of $A \beta$.

Despite the genetic and cell biological evidence that supports the amyloid hypothesis, it is becoming clear that $\mathrm{AD}$ etiology is

Received Aug. 16, 2010; revised Sept. 15, 2010; accepted Sept. 16, 2010.

These studies were supported by National Institutes of Health Grants R01-AG026146 (S.W.P.), P01-AG17617 (R.A.N.), AG008200 and R37 AG017926 (N.K.R.), R01-NS041783 and R01-NS042818 (J.S.), and P01-AG027916 and R01-NS051874 (L.-H.T.). S.W.P., R.A.N., and J.S. also acknowledge funding from the Alzheimer's Association. We thank Dr. Chris Nelson for editing the manuscript.

Correspondence should be addressed to Sanjay W. Pimplikar at the above address. E-mail: pimplis@ccf.org.

DOI:10.1523/JNEUROSCI.4305-10.2010

Copyright $\odot 2010$ the authors $\quad 0270-6474 / 10 / 3014946-09 \$ 15.00 / 0$ complex and that $\mathrm{A} \beta$ alone is unable to account for all aspects of AD (Pimplikar, 2009). For example, recent neuroimaging studies confirm the previous autopsy findings that amyloid deposits are present in cognitively normal individuals, whereas some AD patients show no amyloid deposits in PET (positron emission tomography) scans (Edison et al., 2007; Li et al., 2008). Similarly, it is possible that all of the amyloid-focused clinical trials failed because they were started too late in the disease progression, but the negative outcome is also consistent with the notion that $\mathrm{AD}$ can be caused by $A \beta /$ amyloid-independent factors. The fact that vast overproduction of $A \beta$ peptides in the mouse brain failed to cause neurodegeneration raises further questions as to whether accumulation of $\mathrm{A} \beta$ peptides is indeed the culprit for neurodegeneration in $\mathrm{AD}$. Also, a large number of preclinical studies support roles for calcium dysregulation, proteolysis failure, altered cell signaling, oxidative stress and inflammation in neuronal dysfunction, and neurodegeneration similar to those observed in $\mathrm{AD}$. This article highlights the findings that were presented in a symposium and is not meant to be a comprehensive review of $\mathrm{AD}$ pathogenesis. Here we discuss studies showing that mutations in APP and presenilins can contribute to AD pathology by amyloid-independent mechanisms.

FAD mutations in APP and PS1 lead to defective endo-lysosomal trafficking and proteolysis

The lysosomal network, comprising the endocytic and autophagic pathways, mediates the processing, sorting, and turnover of proteins and other cellular constituents. Endocytosis is especially 


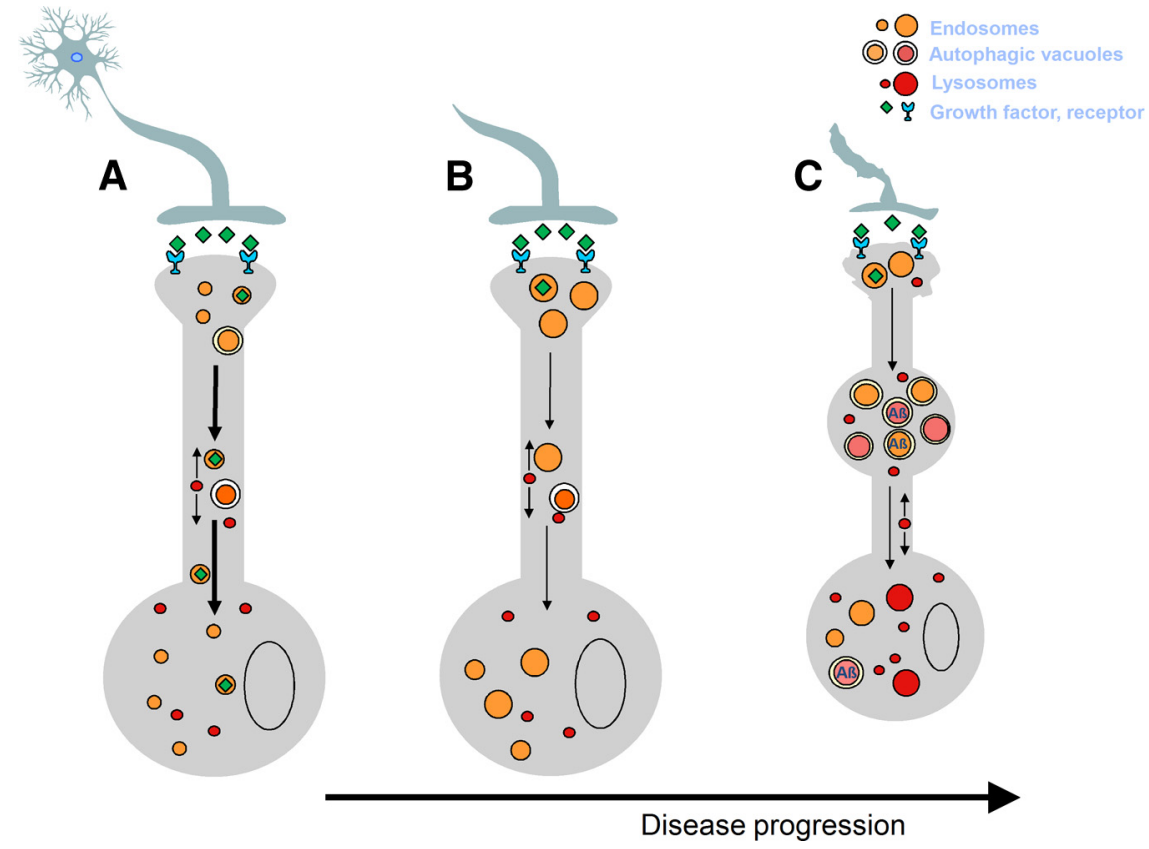

Figure 1. Dysfunction of autophagic and endocytic pathways to lysosomes driven by relevant genes and other risk factors in Alzheimer's disease. $\boldsymbol{A}-\boldsymbol{C}$, A normal neuron is depicted in $\boldsymbol{A}$. At the earliest stages of $\mathrm{AD}(\boldsymbol{B})$, an abnormal acceleration of endocytosis, mediated partly by rab5, is known to be caused by App gene duplication (via $\beta$-CTF) in early onset FAD and Down syndrome and promoted by ApoE4 and elevated cholesterol in late-onset AD. Adverse consequences include endosome enlargement and defective endosome retrograde transport and neurotrophin signaling functions, which promote apoptotic pathway activation and neurodegeneration, particularly of cholinergic neuronal populations. Subsequently $(\boldsymbol{C})$, failure of autophagy, prominently involving impaired lysosomal proteolysis, leads to massive selective accumulation of autophagic vacuoles (autophagosomes, autolysosomes etc,) containing partially digested autophagic and endocytic substrates within swollen "dystrophic" neurites. The diminished clearance by autophagy of toxic organelles and proteins, including $A \beta$, ubiquitinated proteins, activated caspases, and possibly tau, leads to neurodegeneration via multiple pathways. Failure of lysosomal proteolysis and autophagy in $A D$ is driven directly by PS1 mutations in early-onset FAD, and is also promoted by normal aging, oxidative stress, Apo E4, intracellular A $\beta$, and other AD-related genetic and environmental risk factors.

critical in neurons, as it supports such specialized functions as synaptic transmission and retrograde trophic signaling (Nixon et al., 2008). Autophagy, the principal degradative pathway for organelles and long-lived proteins, involves the sequestration of cytoplasmic constituents within autophagosomes followed by digestion of these substrates within autolysosomes that are formed by fusion of autophagosomes with lysosomes. Autophagy is essential for neuronal survival in part by clearing damaged, aggregated, or obsolete proteins in disease states and cellular aging (Wong and Cuervo, 2010). Notably, longevity and cellular aging mechanisms are closely linked to the efficacy of autophagy (Madeo et al., 2010), and during aging, a sine qua non for the development of Alzheimer's disease, autophagy efficiency declines (Cuervo, 2008). The neuron's unique reliance on the lysosomal system is well documented in many primary lysosomal disorders where the defect in a ubiquitous lysosomal protein produces severe neurodegenerative phenotypes (Bellettato and Scarpa, 2010), including pathologies characteristic of $\mathrm{AD}$ (Ohm et al., 2003; Ohmi et al., 2009). The close connection between neurodegeneration and lysosomal system dysfunction is further highlighted by the growing numbers of lysosomal system proteins identified as pathogenic in familial late-onset neurodegenerative disorders, including forms of Parkinson's disease (McCray and Taylor, 2008; Nixon et al., 2008; Cherra et al., 2010).

Recent evidence shows that mutations of PS1 and APP (or $A P P$ gene duplication), independently of $\mathrm{A} \beta$, directly disrupt autophagy or alter endocytosis, which impairs neuronal function and reduces neuron survival (Fig. 1). PS1 has recently been found to be essential for lysosomal proteolysis and autophagy by enabling the acidification of lysosomes required for protease activation (Lee et al., 2010). In PS1-lacking neurons, vacuolar ATPase (vATPase), the proton pump that acidifies lysosomes, is not delivered to lysosomes. Failed lysosome acidification blocks substrate proteolysis during autophagy, causing incompletely degraded proteins to accumulate in autolysosomes. The underlying mechanism involves a novel role for PS1, independent of $\gamma$-secretase, in which PS1 holoprotein, before being cleaved and assembled into $\gamma$-secretase, facilitates $N$-glycosylation of the V01A subunit of vATPase in the endoplasmic reticulum, which is required for its efficient delivery to lysosomes and for assembly of the proton pump. In familial early-onset AD, PS1 mutations lead to a similar loss of lysosome function by the same mechanism (Lee et al., 2010), most likely resulting from a dominant-negative effect of mutant PS1. Deficient lysosomal proteolysis leads to the extensive "neuritic dystrophy" of AD (Suzuki and Terry, 1967; Masliah et al., 1993) characterized by grossly swollen neurites packed with autophagic vacuoles containing $\mathrm{A} \beta$ and other incompletely degraded substrates (Nixon et al., 2005; Yu et al., 2005) that are potentially neurotoxic (Yang et al., 2008). This massive "storage" of waste proteins, reminiscent of lysosomal storage diseases, can be experimentally reproduced in neurons by inhibiting lysosomal cathepsin proteolysis (Boland et al., 2008). Interestingly, impaired autophagy in the $\mathrm{AD}$ brain results in $\mathrm{A} \beta$ accumulation in autolysosomes, and this reservoir of intracellular $A \beta$ may exert further toxicity to the lysosome system (Glabe, 2001). Experimental measures may stimulate autophagy restore lysosomal proteolysis to more normal levels have yielded promising therapeutic effects on neuronal function and cognitive performance in mouse models of $\mathrm{AD}$ (Sun et al., 2008; Spilman et al., 2010) and in certain other neurodegenerative diseases (García-Arencibia et al., 2010).

Autophagy deficits in $\mathrm{AD}$ are part of a continuum of lysosomal system deficits, including endocytic abnormalities that may be manifested as the first specific signs of $\mathrm{AD}$ (Cataldo et al., 2000; Jiang et al., 2010; Rothenberg et al., 2010) (Fig. 1). Abnormal acceleration of neuronal endocytosis is evident before amyloid is deposited in the neocortex (Cataldo et al., 1997, 2000). Genes related to endocytosis, such as Rab5, Rab7, and Rab4, are among the first group to be upregulated in $\mathrm{AD}$ (Ginsberg et al., 2010) and are abnormally recruited to endosomes, which progressively enlarge. This pattern is specific for $\mathrm{AD}$ among studied aging-related neurodegenerative diseases and is accelerated by inheritance of the $\varepsilon 4$ allele of APOE (apolipoprotein E), the major genetic risk factor for late-onset $\mathrm{AD}$ (Cataldo et al., 2000). In a form of $\mathrm{AD}$ caused by App gene duplication and in Down syndrome, where a chromosome 21 segment containing App is trisomic, endosome dysfunction can be attributed to the extra copy of App (Cataldo et al., 2003; Salehi et al., 2006; Jiang et al., 2010) and has been linked 
to altered trophic signaling and cholinergic neurodegeneration (Salehi et al., 2006), and activation of apoptotic pathway (Neve et al., 1996). Recently, these effects of increased App dosage were shown to be mediated specifically by the $\beta$-cleaved C-terminal fragment of APP (Jiang et al., 2010), previously known to have neurotoxic properties relevant to $\mathrm{AD}$ (Oster-Granite et al., 1996; Kim et al., 2000; Choi et al., 2001; Mathews et al., 2002; Arbel et al., 2005; Lee et al., 2006).

\section{FAD mutations in APP and PS1 can exert deleterious effects independent of $\mathrm{A} \boldsymbol{\beta}$}

It has been proposed that PS FAD mutations promote neurodegeneration by increasing neurotoxic peptide $A \beta 42$. More recent work, however, shows that many FAD mutants increase neither production of $A \beta 42$ nor the $A \beta 42 / 40$ ratio that has been thought to initiate $\mathrm{AD}$ pathology (Bentahir et al., 2006; Shioi et al., 2007; Batelli et al., 2008). Also, the Swedish mutation of APP increases production of both $A \beta 42$ and $A \beta 40$ but does not change the ratio (Duering et al., 2005). Thus, although some FAD mutations increase $\mathrm{A} \beta 42$ and/or the $\mathrm{A} \beta 42 / 40$ ratio, not all mutations show this phenomenon. Furthermore, $\mathrm{A} \beta$ peptides are normal components of human serum and CSF, and there is little evidence that $A \beta$ is neurotoxic at in vivo concentrations, which are severalfold lower than the concentrations $\mathrm{A} \beta$ is used in in vitro neurotoxicity assays (for review, see Robakis, 2010).

Currently more than 30 FAD mutations have been mapped on the APP gene. Some of these mutations do not change the primary sequence of $A \beta$ while others fall within the $A \beta$ region. $A$ mutation within this region, Glu693Gln, does not increase $A \beta$ production but increases its tendency to form amyloid. Carriers of this mutation develop the fatal syndrome of hereditary cerebral hemorrhage with amyloidosis of Dutch type (HCHWA-D), characterized by recurrent cerebral hemorrhages due to accumulation of amyloid depositions in cerebral blood vessels. These patients are not classified as AD as they are usually not demented. Other APP mutations on residues 692 and 694, however, are associated with $\mathrm{FAD}$, but these increase neither $\mathrm{A} \beta$ production nor the 42/40 ratio. Interestingly, APP mutations of the London type, which cause relatively small increases in $\mathrm{A} \beta$, induce $\mathrm{AD}$ at earlier ages than the Swedish mutation, which causes much higher increases in $A \beta$ than the London mutations. It should be noted that in APP-based mouse models of AD, all products of APP metabolism are increased together with $\mathrm{A} \beta$, and there is evidence that some of these non-A $\beta$ products are neurotoxic (Nalbantoglu et al., 1997; Ghosal et al., 2009; Nikolaev et al., 2009). Thus, behavioral abnormalities of animal models overexpressing APP need to be interpreted with caution, as in addition to $\mathrm{A} \beta$, other APP metabolites may influence the final outcome and contribute to the mechanism(s) of neurodegeneration (Robakis, 2010).

Indeed, as seen above in the case of vacuolar ATPase, there is ample evidence to indicate that PS1 performs $\gamma$-secretaseindependent functions in addition to its role as a catalytic subunit of the $\gamma$-secretase complex. In addition to its role in regulating calcium homeostasis, wild-type PS1 also stimulates the phosphoinositide 3-kinase (PI3K)/Akt and MEK/ERK (MAP kinase kinase/extracellular signal-regulated protein kinase) signaling pathways and promotes cell survival and growth. By contrast, a number of PS1 FAD mutations fail to stimulate the cell survival pathways and interfere with $\gamma$-secretase-independent functions. These observations reveal the presence of additional mechanisms by which FAD mutations in PS1 may promote neurodegeneration and tau hyperphosphorylation (Baki et al., 2004, 2008; Kang et al., 2005; Tu et al., 2006; Dreses-Werringloer et al., 2008).

Recent data show that in addition to the $\gamma$-cleavage of APP, the PS1-secretase complex promotes the $\varepsilon$-cleavage of other transmembrane proteins, including Notch1 receptor, cadherins, APP, and EphB receptors. This cleavage takes place downstream from the $\gamma$-cleavages site resulting in the release of soluble cytosolic peptides containing the intracellular C-terminal fragments (CTFs). Several of these peptides, including APP intracellular domain (AICD), migrate to the nucleus and regulate gene expression (Cao and Sudhof, 2001; Gao and Pimplikar, 2001), while others remain in the cytoplasm where they regulate stability of transcription factors (Kopan and Ilagan, 2004; Marambaud and Robakis, 2005). Interestingly, $\gamma$-cleaved AICD fragment has been shown to exert deleterious effects and recapitulate multiple AD pathological features in a mouse model (Ryan and Pimplikar, 2005; Ghosal et al., 2009, 2010; Vogt et al., 2009), and peptide EphB2/CTF2 generated by the $\varepsilon$-cleavage of EphB2 receptor stimulates phosphorylation of NMDA receptor subunit NR2B (Xu et al., 2009). Recent data show that in contrast to the proposed gain of function, PS1 FAD mutations may inhibit the cleavage of proteins including APP, cadherins, ephrinB, Notch1, and EphB receptors, leading to reduced production of the corresponding CTF peptides (Song et al., 1999; Marambaud et al., 2003; Wiley et al., 2005; Georgakopoulos et al., 2006; Litterst et al., 


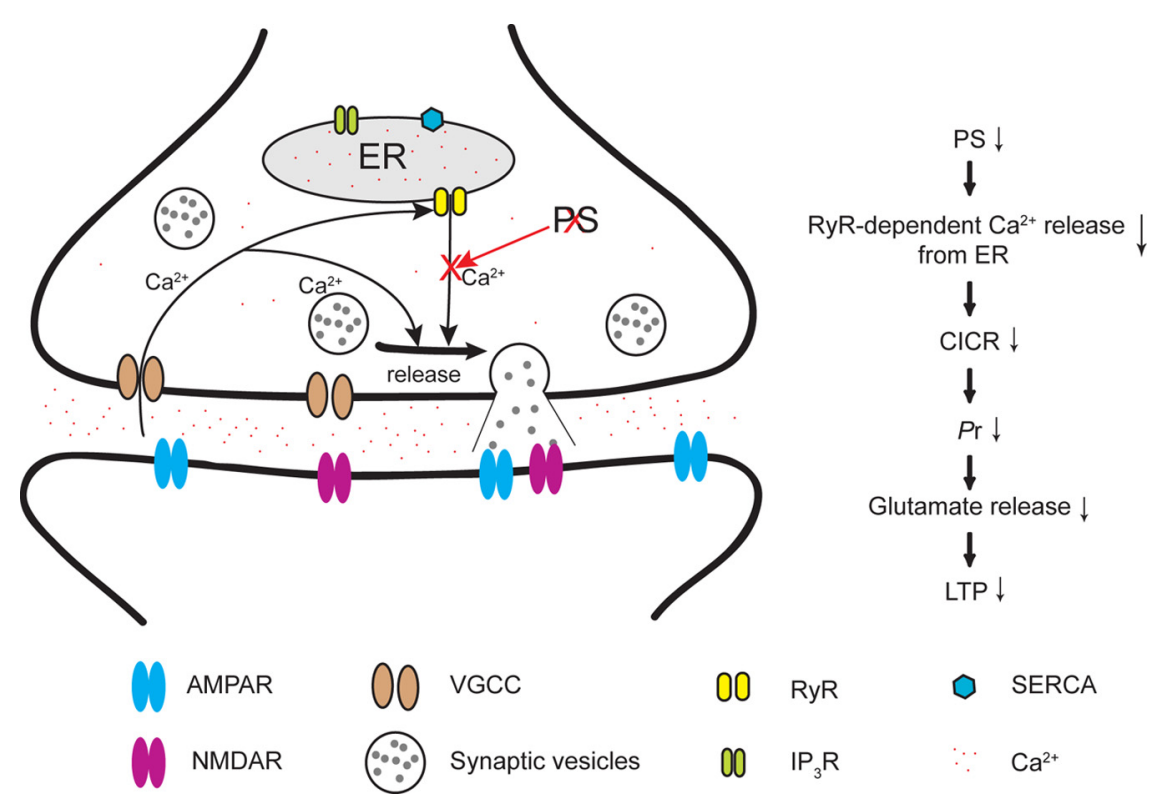

Figure 3. A model depicting the role of presenilins in the regulation of neurotransmitter release. Upon stimulation, calcium concentration at the presynaptic terminal is drastically elevated due to calcium influx through VGCCs and calcium-induced calcium release (CICR) from intracellular stores, which is mediated through both ryanodine receptors and IP, receptors. Loss of PS function in the presynaptic terminal specifically disrupts ryanodine receptor-mediated $\mathrm{Ca}^{2+}$ release from the ER store, thus reducing $\mathrm{CICR}$ and resulting in reduced increases of calcium-induced by action potentials in the presynaptic terminal. This reduction in calcium increases impairs the probability of neurotransmitter release, and the decreased glutamate release causes LTP impairment in PS-deficient presynaptic terminals. (Figure taken from supplementary information in Nature 460:632-636, 2009. Reprinted with permission.)

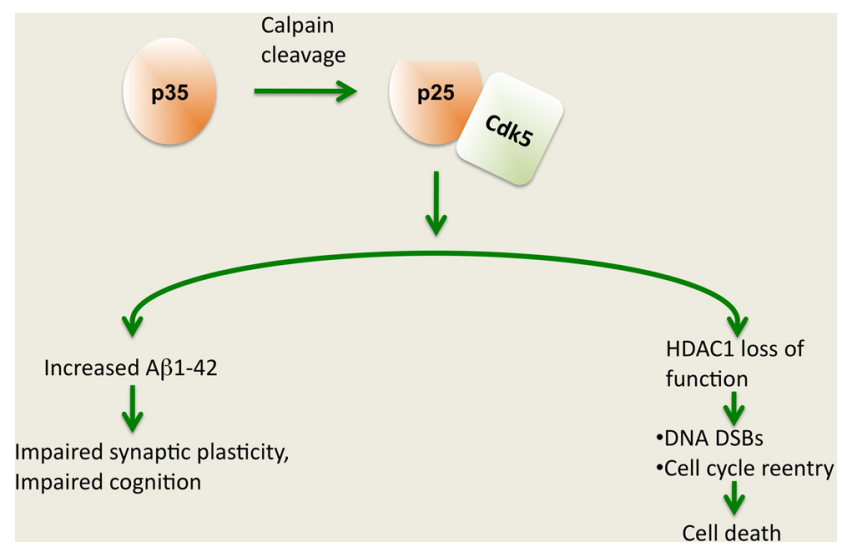

Figure 4. $\quad \mathrm{p} 25 / \mathrm{Cdk} 5$ in the pathogenesis of Alzheimer's disease. The $\mathrm{p} 25 / \mathrm{Cdk} 5$ kinase exerts two parallel processes in the course of neurodegeneration. First, it increases $\beta$-amyloid production which contributes to synaptic impairment and memory loss. Second, p25/Cdk5 in the nucleus reduces $\mathrm{HDAC} 1$ activity, which leads to increased expression of cell cycle genes and DNA double-strand breaks. These pathologies eventually lead to neuronal loss and neurodegeneration. DSBs, double-strand breaks.

2007). These data raise the possibility (Fig. 2) that FAD mutations promote neurodegeneration by altering the production of peptides with important transcriptional and signal transduction properties (Robakis, 2003).

The autosomal-dominant mode of FAD transmission is consistent with the notion that these mutations cause gain of a toxic function. However, there is a possibility that some FAD mutations in PS1 may function through allelic interference. Evidence that PS1 FAD mutants inhibit the $\gamma$-secretase-catalyzed $\varepsilon$-cleavages of many substrates (loss of function, see above), combined with absence of FAD haploinsufficiency mutants, raises the possibility of a model of allelic interference in which products of inactive FAD mutant alleles of PS promote autosomal-dominant neurodegeneration by also inhibiting the functions of the wild-type protein (Robakis, 2010).

\section{Synaptic dysfunction and neuronal degeneration caused by loss of PS}

The presenilin genes harbor most of the FAD-linked mutations and are highly expressed in pyramidal neurons of the adult cerebral cortex, where $\mathrm{AD}$ pathogenesis manifests. Analysis of viable presenilin conditional knock-out mice, in which presenilin expression is selectively inactivated in excitatory pyramidal neurons of the postnatal forebrain, revealed important PS functions relevant to AD pathogenesis (Yu et al., 2001; Saura et al., 2004). Specifically, loss of presenilins affects both short- and long-term plasticity, in the absence of neurodegeneration. Furthermore, NMDA receptor-mediated responses are impaired and synaptic levels of NMDA receptor subunits are reduced in the absence of PS. Interestingly, loss of PS reduces levels of cAMP response element-binding protein (CREB)-binding protein (CBP) and transcription of CREB/CBP target genes (Saura et al., 2004; Beglopoulos and Shen, 2006), even though it was subsequently observed that CREB-mediated transcription is regulated indirectly by PS (Watanabe et al., 2009). Strikingly, PS cDKO mice (conditional double knock-out mice lacking both presenilins in the postnatal forebrain) develop in an age-dependent manner synaptic, dendritic, and neuronal degeneration with accompanying astrogliosis and hyperphosphorylation of tau, demonstrating an essential role for PS in neuronal survival (Beglopoulos et al., 2004; Saura et al., 2004; Wines-Samuelson et al., 2010). Specifically, while presenilins are inactivated at 4 weeks of age postnatally in $P S \mathrm{cDKO}$ mice, significant increases $(\sim 8$-fold, compared with control mice) of apoptotic cell death are first detected at 2 months of age. However, this represents only $\sim 0.1 \%$ of cortical neurons that are undergoing apoptosis; thus, the total cortical neuron number and volume are not significantly altered at this age. By 4 months of age, $\sim 9 \%$ of cortical neurons are lost in PS cDKO mice, followed by $18 \%$ and $24 \%$ neuronal loss at 6 and 9 months of age, respectively. Furthermore, presenilins appear to promote memory and neuronal survival in a $\gamma$-secretase-dependent manner, as conditional inactivation of nicastrin, another component of the $\gamma$-secretase complex, in the adult cerebral cortex similarly resulted in progressive memory impairment and neurodegeneration (Tabuchi et al., 2009). These in vivo findings and a large number of reports on the effects of FAD-linked mutations in culture and in vitro systems as well as in Caenorhabditis elegans raised the possibility that PS mutations may cause dementia and neurodegeneration in $\mathrm{AD}$ via a partial loss-of-function and dominant-negative mechanism (Shen and Kelleher, 2007). Indeed, a recent report showed that pathogenic mutations in PS1, such as L435F, could result in complete loss of $\gamma$-secretase activity (Heilig et al., 2010). The later onset of the disease in AD patients carrying presenilin mutations, compared with $P S$ cDKO mouse models, can be explained by the fact that while FAD mutations 
confer partial (or complete) loss of presenilin activity, only one of the $P S$ alleles is affected in FAD patients.

The fact that synaptic impairments precede progressive neurodegeneration suggests that synaptic dysfunction caused by loss of PS function promotes subsequent neuronal degeneration. The role of presenilins in the synapse was elucidated by systematic genetic analysis through the restriction of presenilin inactivation to hippocampal CA1 or CA3 neurons (Zhang et al., 2009). This strategy permitted analysis of the effects of presenilin inactivation in either presynaptic or postsynaptic neurons of the Schaeffer collateral pathway. It was found that long-term potentiation (LTP) induced by theta burst stimulation is decreased after presynaptic but not postsynaptic deletion of presenilins. Moreover, presynaptic but not postsynaptic inactivation of presenilins impairs short-term plasticity and synaptic facilitation, and the defects in synaptic facilitation are dependent upon the frequency used for stimulation and the external calcium concentration. The probability of evoked glutamate release, measured by the decay curve of the open-channel NMDA receptor antagonist MK-801 [5Hdibenzo $[a, d]$ cyclohepten-5,10-imine (dizocilpine maleate)], is reduced by presynaptic inactivation of presenilins. To explore further the involvement of calcium in the presynaptic defects caused by loss of presenilins, both calcium influx and efflux were evaluated, and it was found that the current-voltage relationship of voltage-gated calcium current is normal in the absence of PS. Strikingly, depletion of calcium internal stores by thapsigargin, a noncompetitive SERCA (sarcoplasmic/endoplasmic reticulum calcium ATPase) calcium pump, mimics and occludes the effects of presynaptic presenilin inactivation, suggesting a defect in calcium influx underlying the presynaptic impairment. Blockade of calcium release from ryanodine receptors using two independent inhibitors had effects similar to those of thapsigargin, whereas blockers of $\mathrm{IP}_{3}$ receptors had no effect on presynaptic frequency facilitation. These findings were further corroborated using dissociated PS-null hippocampal neuronal cultures from postnatal pups, in which the requirement of presenilins in neural development is circumvented. These cultured $P S$-null hippocampal neurons show normal neuronal morphology and synaptic density and recapitulate the presynaptic defects seen in acute hippocampal slices of CA3-PS CDKO mice, suggesting that they are relevant experimental systems to assess calcium homeostasis directly. Indeed, depolarization-induced calcium increases in the cytosol, which are composed of both calcium influx and calcium-induced calcium release from intracellular stores, are reduced in hippocampal neurons lacking both presenilins. Furthermore, blockade of ryanodine receptors but not $\mathrm{IP}_{3}$ receptors mimics and occludes the effects of presenilin inactivation.

Collectively, these genetic and electrophysiological studies demonstrated that loss of presenilin function impairs LTP induction and glutamatergic neurotransmitter release by a presynaptic mechanism (Fig. 3). These findings raised the possibility that presynaptic mechanisms may play a primary role in $\mathrm{AD}$ pathophysiology (Shen, 2010). In support of this hypothesis, presenilins are localized to presynaptic terminals (Zhang et al., 2009) and APP C-terminal fragments, precursors of $\mathrm{A} \beta$, accumulate in presynaptic terminals of PS1 cKO mice (Saura et al., 2005). A time course study to identify temporal development of presynaptic and postsynaptic defects in forebrain-PS cDKO mice further revealed that presynaptic defects, such as synaptic facilitation, occur at 5 weeks of age, followed by postsynaptic defects, such as NMDA receptor-mediated responses, at 6 weeks of age, providing additional support for the importance of presynaptic roles played by presenilins (Zhang et al., 2010).

\section{DNA damage by aberrantly activated cyclin-dependent}

\section{kinase 5}

Cyclin-dependent kinase 5 (Cdk5) is a proline-directed serine/ threonine kinase that has important roles in various neuronal functions including brain development, synaptogenesis, synaptic plasticity, and memory formation (Dhavan and Tsai, 2001). Cdk5 is activated when bound to one of its two activators, p35 or p39, which have related primary sequences. Aberrant activation of Cdk5 can occur through the proteolytic cleavage of p35 to p25 via calpain, a calcium-dependent protease (Kusakawa et al., 2000; Lee et al., 2000; Nath et al., 2000). Evidence for a role of Cdk5 in 
the pathogenesis of neurodegenerative conditions has been accumulating over the last several years (Patrick et al., 1999; Wang et al., 2003; Qu et al., 2007).

Cdk5 activity is increased in postmortem AD brains; this is likely due to increased p25 levels found in AD brain tissues compared with age-matched control brain tissues (Patrick et al., 1999). Levels of p 25 are also increased in AD mouse models, including the PS1-deficient and 5XFAD mice (Oakley et al., 2006). In vitro, $\mathrm{A} \beta 1-42$ causes $\mathrm{p} 25$ production in primary dissociated neurons (Lee et al., 2000). A forebrain-specific inducible p25 (CK-p25) transgenic mouse model exhibits elevated $A \beta 1-42$ peptide, neurodegeneration characterized by massive neuronal and synaptic loss, tau-associated pathology, and learning and memory impairments (Cruz et al., 2003; Fischer et al., 2005; Kim et al., 2008). Inhibition of Cdk5 activity in transgenic $\mathrm{p} 25$ mice reduces $\mathrm{A} \beta 1-42$ production suggesting that $A \beta 1-42$ processing is regulated by the $C d k 5 / p 25$ complex (Wen et al., 2008). Conversely, inhibition of $\mathrm{A} \beta$ generation by BACE1 loss of function in the CK-p 25 mice rescued the impairments in synaptic plasticity and memory formation (L.H.T., unpublished observation). These results indicate that $\mathrm{A} \beta$ plays a crucial role in neurodegeneration exhibited by the CK-p25 mice. Furthermore, they hint at the possibility that p25 and A $\beta$ collaborate to cause neuronal death.

To decipher the cellular mechanism(s) that leads to neurodegeneration, microarray gene expression profiling experiments were performed in CK-p25 mice before the detection of astrogliosis and neuronal loss (Kim et al., 2008). Surprisingly, two main classes of genes were profoundly upregulated in the brain of CK-p25 mice compared with control mice. The first class is cellular proteins known to participate in mitotic cell division including cyclins, mitotic Cdks, proliferating cell nuclear antigen, and E2F. The second class is components of the DNA damage response pathway, especially DNA double-stranded break, including RAD51, ATM, and DNA polymerase $\varepsilon$. Indeed, reactivation of cell cycle genes and DNA double-stranded break lesions were demonstrated in the hippocampal neurons of CK-p25 mice before the onset of neurodegeneration indicating that these events may cause neuronal loss. Reactivation of cell cycle genes has been reported in postmortem AD patients. Preliminary data indicate that the numbers of neurons with DNA double-stranded break are significantly increased in $\mathrm{AD}$ brains (L.H.T., unpublished observations).

It was postulated that a common mechanism may underlie both cell cycle reactivation and DNA double-stranded breaks, as the two events were largely observed in the same neurons in CK-p25 mice. Histone deacetylase 1 (HDAC1) has been shown to repress the expression of numerous cell cycle proteins by binding to their upstream regulatory elements (Brehm et al., 1998; Stiegler et al., 1998; Lagger et al., 2002; Rayman et al., 2002). In CK-p25 mice, HDAC1 activity was downregulated, and its association with the genes encoding cell cycle proteins as well as with chromatin was reduced. Furthermore, HDAC1 loss of function caused DNA double-stranded breaks, reactivation of cell cycle genes, and neuronal death. Conversely, HDAC1 gain of function ameliorated p25-induced DNA damage and cell death. Thus, reduced HDAC1 activity resulting from p25 accumulation may contribute to several early events leading to neurodegeneration.

In conclusion, we think that $\mathrm{p} 25 / \mathrm{Cdk} 5$ concurrently exerts two parallel processes within the neuron (Fig. 4). One aspect involves deficits in synaptic plasticity, and learning and memory in which $\mathrm{p} 25 / \mathrm{Cdk} 5$ leads to increased $\beta$-amyloid, which in turn contributes to synaptic impairments and memory loss.
Meanwhile, p25/Cdk5 also exerts nuclear activity, resulting in dysregulation of HDAC1. HDAC1 loss of function causes the upregulation of cell cycle genes and DNA double-strand breaks. These pathologies eventually lead to neuronal loss and neurodegeneration. Our results suggest that p25 generation plays a very upstream and critical role in the signaling cascade leading to neurodegeneration, contributing to both cognitive symptoms and cellular demise. Thus, targeting Cdk5 or p25 generation may provide new promising avenues for therapeutic intervention of Alzheimer's disease.

\section{Concluding remarks}

Today, research in the field of $\mathrm{AD}$ is on the precipice of a crucial and somewhat paradoxical juncture. On the one hand, progress made in multiple fields of investigation (genetics, biochemistry, cell biology, epidemiology, neuroimaging, etc.) has yielded tremendous insights into the possible cause of $\mathrm{AD}$, and we are closer to finding a disease-modifying treatment. On the other hand, it has become increasingly clear that disproportionate reliance on $\mathrm{A} \beta$ /amyloid-based mechanisms to explain $\mathrm{AD}$ etiology and nearly exclusive emphasis on amyloid as a therapeutic target have not yielded the desired results. Genetic studies of FAD, which accounts for $\sim 3-5 \%$ of $\mathrm{AD}$ cases, have been considered the strongest evidence supporting the amyloid hypothesis; however, as discussed above, there is increasing evidence that even the FAD mutations in APP and presenilins can act via amyloid-independent mechanisms. Indeed, it is clear from the data discussed above that the amyloiddependent and amyloid-independent mechanisms are not mutually exclusive and both can contribute to $\mathrm{AD}$ pathology.

An important challenge for future studies will be to determine the extent to which amyloid-independent mechanisms contribute to $\mathrm{AD}$. The implicit assumption underlying the current drug trials is that the prime causative agent of $\mathrm{AD}$ is amyloid/A $\beta$ peptides, and therefore that blocking amyloid accumulation will prevent $\mathrm{AD}$. However, if amyloid-independent mechanisms also make significant contributions to $\mathrm{AD}$, then the current drug trials will yield only moderately positive results, as the case has been. Another challenge facing future investigations will be to produce a satisfactory explanation as to why $\mathrm{AD}$ takes so long to manifest itself and why certain parts of the brain (limbic system) are more susceptible in $\mathrm{AD}$. As future studies seek answers to these questions, it is becoming clear that both amyloid-dependent and amyloid-independent mechanisms (Fig. 5) are involved in contributing to $\mathrm{AD}$ pathology and that an effective diseasemodifying treatment will arise only from a strategy that addresses both of these mechanisms.

\section{References}

Arbel M, Yacoby I, Solomon B (2005) Inhibition of amyloid precursor protein processing by beta-secretase through site-directed antibodies. Proc Natl Acad Sci U S A 102:7718-7723.

Baki L, Shioi J, Wen P, Shao Z, Schwarzman A, Gama-Sosa M, Neve R, Robakis NK (2004) PS1 activates PI3K thus inhibiting GSK-3 activity and tau overphosphorylation: effects of FAD mutations. EMBO J 23:2586-2596.

Baki L, Neve RL, Shao Z, Shioi J, Georgakopoulos A, Robakis NK (2008) Wild-type but not FAD mutant presenilin-1 prevents neuronal degeneration by promoting phosphatidylinositol 3-kinase neuroprotective signaling. J Neurosci 28:483-490.

Batelli S, Albani D, Prato F, Polito L, Franceschi M, Gavazzi A, Forloni G (2008) Early-onset Alzheimer disease in an Italian family with presenilin-1 double mutation E318G and G394V. Alzheimer Dis Assoc Disord 22:184-187.

Beglopoulos V, Shen J (2006) Regulation of CRE-dependent transcription by presenilins: prospects for therapy of Alzheimer's disease. Trends Pharmacol Sci 27:33-40. 
Beglopoulos V, Sun X, Saura CA, Lemere CA, Kim RD, Shen J (2004) Reduced beta-amyloid production and increased inflammatory responses in presenilin conditional knock-out mice. J Biol Chem 279:46907-46914.

Bellettato CM, Scarpa M (2010) Pathophysiology of neuropathic lysosomal storage disorders. J Inherit Metab Dis 33:347-362.

Bentahir M, Nyabi O, Verhamme J, Tolia A, Horre K, Wiltfang J, Esselmann H, De Strooper B (2006) Presenilin clinical mutations can affect gammasecretase activity by different mechanisms. J Neurochem 96:732-742.

Boland B, Kumar A, Lee S, Platt FM, Wegiel J, Yu WH, Nixon RA (2008) Autophagy induction and autophagosome clearance in neurons: relationship to autophagic pathology in Alzheimer's disease. J Neurosci 28:6926-6937.

Brehm A, Miska EA, McCance DJ, Reid JL, Bannister AJ, Kouzarides T (1998) Retinoblastoma protein recruits histone deacetylase to repress transcription. Nature 391:597-601.

Cao X, Sudhof TC (2001) A transcriptionally [correction of transcriptively] active complex of APP with Fe65 and histone acetyltransferase Tip60. Science 293:115-120.

Cataldo AM, Barnett JL, Pieroni C, Nixon RA (1997) Increased neuronal endocytosis and protease delivery to early endosomes in sporadic Alzheimer's disease: neuropathologic evidence for a mechanism of increased beta-amyloidogenesis. J Neurosci 17:6142-6151.

Cataldo AM, Peterhoff CM, Troncoso JC, Gomez-Isla T, Hyman BT, Nixon RA (2000) Endocytic pathway abnormalities precede amyloid beta deposition in sporadic Alzheimer's disease and Down syndrome: differential effects of APOE genotype and presenilin mutations. Am J Pathol 157:277-286.

Cataldo AM, Petanceska S, Peterhoff CM, Terio NB, Epstein CJ, Villar A, Carlson EJ, Staufenbiel M, Nixon RA (2003) App gene dosage modulates endosomal abnormalities of Alzheimer's disease in a segmental trisomy 16 mouse model of down syndrome. J Neurosci 23:6788-6792.

Cherra SJ 3rd, Dagda RK, Chu CT (2010) Review: autophagy and neurodegeneration: survival at a cost? Neuropathol Appl Neurobiol 36:125-132.

Choi SH, Park CH, Koo JW, Seo JH, Kim HS, Jeong SJ, Lee JH, Kim SS, Suh YH (2001) Memory impairment and cholinergic dysfunction by centrally administered Abeta and carboxyl-terminal fragment of Alzheimer's APP in mice. FASEB J 15:1816-1818.

Cruz JC, Tseng HC, Goldman JA, Shih H, Tsai L-H (2003) Aberrant Cdk5 activation by $\mathrm{p} 25$ triggers pathological events leading to neurodegeneration and neurofibrillary tangles. Neuron 40:471-483.

Cuervo AM (2008) Autophagy and aging: keeping that old broom working. Trends Genet 24:604-612.

Dhavan R, Tsai LH (2001) A decade of CDK5. Nat Rev Mol Cell Biol 2:749-759.

Dreses-Werringloer U, Lambert JC, Vingtdeux V, Zhao H, Vais H, Siebert A, Jain A, Koppel J, Rovelet-Lecrux A, Hannequin D, Pasquier F, Galimberti D, Scarpini E, Mann D, Lendon C, Campion D, Amouyel P, Davies P, Foskett JK, Campagne F, et al. (2008) A polymorphism in CALHM1 influences $\mathrm{Ca} 2+$ homeostasis, Abeta levels, and Alzheimer's disease risk. Cell 133:1149-1161.

Duering M, Grimm MO, Grimm HS, Schröder J, Hartmann T (2005) Mean age of onset in familial Alzheimer's disease is determined by amyloid beta 42. Neurobiol Aging 26:785-788.

Edison P, Archer HA, Hinz R, Hammers A, Pavese N, Tai YF, Hotton G, Cutler D, Fox N, Kennedy A, Rossor M, Brooks DJ (2007) Amyloid, hypometabolism, and cognition in Alzheimer disease: an $\left[{ }^{11} \mathrm{C}\right] \mathrm{PIB}$ and $\left[{ }^{18}\right.$ F]FDG PET study. Neurology 68:501-508.

Fischer A, Sananbenesi F, Pang PT, Lu B, Tsai LH (2005) Opposing roles of transient and prolonged expression of p25 in synaptic plasticity and hippocampus-dependent memory. Neuron 48:825-838.

Gao Y, Pimplikar SW (2001) The gamma-secretase-cleaved C-terminal fragment of amyloid precursor protein mediates signaling to the nucleus. Proc Natl Acad Sci U S A 98:14979-14984.

García-Arencibia M, Hochfeld WE, Toh PP, Rubinsztein DC (2010) Autophagy, a guardian against neurodegeneration. Semin Cell Dev Biol 21:691-698.

Georgakopoulos A, Litterst C, Ghersi E, Baki L, Xu C, Serban G, Robakis NK (2006) Metalloproteinase/presenilin1 processing of ephrinB regulates EphB-induced Src phosphorylation and signaling. EMBO J 25:12421252.
Ghosal K, Vogt DL, Liang M, Shen Y, Lamb BT, Pimplikar SW (2009) Alzheimer's disease-like pathological features in transgenic mice expressing the APP intracellular domain. Proc Natl Acad Sci U S A 106:1836718372.

Ghosal K, Stathopoulos A, Pimplikar SW (2010) APP intracellular domain impairs adult neurogenesis in transgenic mice by inducing neuroinflammation. PLoS One 5:e11866.

Ginsberg SD, Alldred MJ, Counts SE, Cataldo AM, Neve RL, Jiang Y, Wuu J, Chao MV, Mufson EJ, Nixon RA, Che S (2010) Microarray analysis of hippocampal CA1 neurons implicates early endosomal dysfunction during Alzheimer's disease progression. Biol Psychiatry 68:885-893.

Glabe C (2001) Intracellular mechanisms of amyloid accumulation and pathogenesis in Alzheimer's disease. J Mol Neurosci 17:137-145.

Hardy J, Selkoe DJ (2002) The amyloid hypothesis of Alzheimer's disease: progress and problems on the road to therapeutics. Science 297:353-356.

Heilig EA, Xia W, Shen J, Kelleher RJ 3rd (2010) A presenilin-1 mutation identified in familial Alzheimer disease with cotton wool plaques causes a nearly complete loss of gamma-secretase activity. J Biol Chem 285:22350-22359.

Jiang Y, Mullaney KA, Peterhoff CM, Che S, Schmidt SD, Boyer-Boiteau A, Ginsberg SD, Cataldo AM, Mathews PM, Nixon RA (2010) Alzheimer'srelated endosome dysfunction in Down syndrome is Abeta-independent but requires APP and is reversed by BACE-1 inhibition. Proc Natl Acad Sci U S A 107:1630-1635.

Kang DE, Yoon IS, Repetto E, Busse T, Yermian N, Ie L, Koo EH (2005) Presenilins mediate phosphatidylinositol 3-kinase/AKT and ERK activation via select signaling receptors. Selectivity of PS2 in platelet-derived growth factor signaling. J Biol Chem 280:31537-31547.

Kim D, Frank CL, Dobbin MM, Tsunemoto RK, Tu W, Peng PL, Guan JS, Lee BH, Moy LY, Giusti P, Broodie N, Mazitschek R, Delalle I, Haggarty SJ, Neve RL, Lu Y, Tsai LH (2008) Deregulation of HDAC1 by p25/Cdk5 in neurotoxicity. Neuron 60:803-817.

Kim HS, Park CH, Cha SH, Lee JH, Lee S, Kim Y, Rah JC, Jeong SJ, Suh YH (2000) Carboxyl-terminal fragment of Alzheimer's APP destabilizes calcium homeostasis and renders neuronal cells vulnerable to excitotoxicity. FASEB J 14:1508-1517.

Kopan R, Ilagan MX (2004) Gamma-secretase: proteasome of the membrane? Nat Rev Mol Cell Biol 5:499-504.

Kusakawa G, Saito T, Onuki R, Ishiguro K, Kishimoto T, Hisanaga S (2000) Calpain-dependent proteolytic cleavage of the p35 cyclin-dependent kinase 5 activator to p25. J Biol Chem 275:17166-17172.

Lagger G, O'Carroll D, Rembold M, Khier H, Tischler J, Weitzer G, Schuettengruber B, Hauser C, Brunmeir R, Jenuwein T, Seiser C (2002) Essential function of histone deacetylase 1 in proliferation control and CDK inhibitor repression. EMBO J 21:2672-2681.

Lee JH, Yu WH, Kumar A, Lee S, Mohan PS, Peterhoff CM, Wolfe DM, Martinez-Vicente M, Massey AC, Sovak G, Uchiyama Y, Westaway D, Cuervo AM, Nixon RA (2010) Lysosomal proteolysis and autophagy require presenilin 1 and are disrupted by Alzheimer-related PS1 mutations. Cell 141:1146-1158.

Lee KW, Im JY, Song JS, Lee SH, Lee HJ, Ha HY, Koh JY, Gwag BJ, Yang SD, Paik SG, Han PL (2006) Progressive neuronal loss and behavioral impairments of transgenic C57BL/6 inbred mice expressing the carboxy terminus of amyloid precursor protein. Neurobiol Dis 22:10-24.

Lee MS, Kwon YT, Li M, Peng J, Friedlander RM, Tsai LH (2000) Neurotoxicity induces cleavage of p35 to p25 by calpain. Nature 405:360-364.

Li Y, Rinne JO, Mosconi L, Pirraglia E, Rusinek H, DeSanti S, Kemppainen N, Någren K, Kim BC, Tsui W, de Leon MJ (2008) Regional analysis of FDG and PIB-PET images in normal aging, mild cognitive impairment, and Alzheimer's disease. Eur J Nucl Med Mol Imaging 35:2169-2181.

Litterst C, Georgakopoulos A, Shioi J, Ghersi E, Wisniewski T, Wang R, Ludwig A, Robakis NK (2007) Ligand binding and calcium influx induce distinct ectodomain/gamma-secretase-processing pathways of EphB2 receptor. J Biol Chem 282:16155-16163.

Madeo F, Tavernarakis N, Kroemer G (2010) Can autophagy promote longevity? Nat Cell Biol 12:842-846.

Marambaud P, Robakis NK (2005) Genetic and molecular aspects of Alzheimer's disease shed light on new mechanisms of transcriptional regulation. Genes Brain Behav 4:134-146.

Marambaud P, Wen PH, Dutt A, Shioi J, Takashima A, Siman R, Robakis NK 
(2003) A CBP binding transcriptional repressor produced by the PS1/ epsilon-cleavage of $\mathrm{N}$-cadherin is inhibited by PS1 FAD mutations. Cell 114:635-645.

Masliah E, Mallory M, Deerinck T, DeTeresa R, Lamont S, Miller A, Terry RD, Carragher B, Ellisman M (1993) Re-evaluation of the structural organization of neuritic plaques in Alzheimer's disease. J Neuropathol Exp Neurol 52:619-632.

Mathews PM, Jiang Y, Schmidt SD, Grbovic OM, Mercken M, Nixon RA (2002) Calpain activity regulates the cell surface distribution of amyloid precursor protein. Inhibition of calpains enhances endosomal generation of beta-cleaved C-terminal APP fragments. J Biol Chem 277:3641536424.

McCray BA, Taylor JP (2008) The role of autophagy in age-related neurodegeneration. Neurosignals 16:75-84.

Nalbantoglu J, Tirado-Santiago G, Lahsaïni A, Poirier J, Goncalves O, Verge G, Momoli F, Welner SA, Massicotte G, Julien JP, Shapiro ML (1997) Impaired learning and LTP in mice expressing the carboxy terminus of the Alzheimer amyloid precursor protein. Nature 387:500-505.

Nath R, Davis M, Probert AW, Kupina NC, Ren X, Schielke GP, Wang KK (2000) Processing of cdk5 activator p35 to its truncated form (p25) by calpain in acutely injured neuronal cells. Biochem Biophys Res Commun 274:16-21.

Neve RL, Boyce FM, McPhie DL, Greenan J, Oster-Granite ML (1996) Transgenic mice expressing APP-C100 in the brain. Neurobiol Aging 17:191-203.

Nikolaev A, McLaughlin T, O’Leary DD, Tessier-Lavigne M (2009) APP binds DR6 to trigger axon pruning and neuron death via distinct caspases. Nature 457:981-989.

Nixon RA, Wegiel J, Kumar A, Yu WH, Peterhoff C, Cataldo A, Cuervo AM (2005) Extensive involvement of autophagy in Alzheimer disease: an immuno-electron microscopy study. J Neuropathol Exp Neurol 64:113-122.

Nixon RA, Yang DS, Lee JH (2008) Neurodegenerative lysosomal disorders: a continuum from development to late age. Autophagy 4:590-599.

Oakley H, Cole SL, Logan S, Maus E, Shao P, Craft J, Guillozet-Bongaarts A, Ohno M, Disterhoft J, Van Eldik L, Berry R, Vassar R (2006) Intraneuronal beta-amyloid aggregates, neurodegeneration, and neuron loss in transgenic mice with five familial Alzheimer's disease mutations: potential factors in amyloid plaque formation. J Neurosci 26:10129-10140.

Ohm TG, Treiber-Held S, Distl R, Glockner F, Schonheit B, Tamanai M, Meske V (2003) Cholesterol and tau protein-findings in Alzheimer's and Niemann Pick C's disease. Pharmacopsychiatry 36 [Suppl 2]:S120-S126.

Ohmi K, Kudo LC, Ryazantsev S, Zhao HZ, Karsten SL, Neufeld EF (2009) Sanfilippo syndrome type B, a lysosomal storage disease, is also a tauopathy. Proc Natl Acad Sci U S A 106:8332-8337.

Oster-Granite ML, McPhie DL, Greenan J, Neve RL (1996) Age-dependent neuronal and synaptic degeneration in mice transgenic for the $\mathrm{C}$ terminus of the amyloid precursor protein. J Neurosci 16:6732-6741.

Patrick GN, Zukerberg L, Nikolic M, de la Monte S, Dikkes P, Tsai LH (1999) Conversion of p35 to p 25 deregulates Cdk5 activity and promotes neurodegeneration. Nature 402:615-622.

Pimplikar SW (2009) Reassessing the amyloid cascade hypothesis of Alzheimer's disease. Int J Biochem Cell Biol 41:1261-1268.

Price DL, Tanzi RE, Borchelt DR, Sisodia SS (1998) Alzheimer's disease: genetic studies and transgenic models. Annu Rev Genet 32:461-493.

Qu D, Rashidian J, Mount MP, Aleyasin H, Parsanejad M, Lira A, Haque E, Zhang Y, Callaghan S, Daigle M, Rousseaux MW, Slack RS, Albert PR, Vincent I, Woulfe JM, Park DS (2007) Role of Cdk5-mediated phosphorylation of Prx2 in MPTP toxicity and Parkinson's disease. Neuron 55:37-52.

Rayman JB, Takahashi Y, Indjeian VB, Dannenberg JH, Catchpole S, Watson RJ, te Riele H, Dynlacht BD (2002) E2F mediates cell cycle-dependent transcriptional repression in vivo by recruitment of an $\mathrm{HDAC} 1 / \mathrm{mSin} 3 \mathrm{~B}$ corepressor complex. Genes Dev 16:933-947.

Robakis NK (2003) An Alzheimer's disease hypothesis based on transcriptional dysregulation. Amyloid 10:80-85.

Robakis NK (2010) Mechanisms of AD neurodegeneration may be independent of Abeta and its derivatives. Neurobiol Aging. Advance online publication. Retrieved Nov. 1, 2010. doi:10.1016/j.nerobiolaging.2010.05.022.

Rothenberg C, Srinivasan D, Mah L, Kaushik S, Peterhoff CM, Ugolino J,
Fang S, Cuervo AM, Nixon RA, Monteiro MJ (2010) Ubiquilin functions in autophagy and is degraded by chaperone-mediated autophagy. Hum Mol Genet 19:3219-3232.

Ryan KA, Pimplikar SW (2005) Activation of GSK-3 and phosphorylation of CRMP2 in transgenic mice expressing APP intracellular domain. J Cell Biol 171:327-335.

Salehi A, Delcroix JD, Belichenko PV, Zhan K, Wu C, Valletta JS, TakimotoKimura R, Kleschevnikov AM, Sambamurti K, Chung PP, Xia W, Villar A, Campbell WA, Kulnane LS, Nixon RA, Lamb BT, Epstein CJ, Stokin GB, Goldstein LS, Mobley WC (2006) Increased App expression in a mouse model of Down's syndrome disrupts NGF transport and causes cholinergic neuron degeneration. Neuron 51:29-42.

Saura CA, Choi SY, Beglopoulos V, Malkani S, Zhang D, Shankaranarayana Rao BS, Chattarji S, Kelleher RJ 3rd, Kandel ER, Duff K, Kirkwood A, Shen J (2004) Loss of presenilin function causes impairments of memory and synaptic plasticity followed by age-dependent neurodegeneration. Neuron 42:23-36.

Saura CA, Chen G, Malkani S, Choi SY, Takahashi RH, Zhang D, Gouras GK, Kirkwood A, Morris RG, Shen J (2005) Conditional inactivation of presenilin 1 prevents amyloid accumulation and temporarily rescues contextual and spatial working memory impairments in amyloid precursor protein transgenic mice. J Neurosci 25:6755-6764.

Shen J (2010) Impaired neurotransmitter release in Alzheimer's and Parkinson's diseases. Neurodegener Dis 7:80-83.

Shen J, Kelleher RJ 3rd (2007) The presenilin hypothesis of Alzheimer's disease: evidence for a loss-of-function pathogenic mechanism. Proc Natl Acad Sci U S A 104:403-409.

Shioi J, Georgakopoulos A, Mehta P, Kouchi Z, Litterst CM, Baki L, Robakis NK (2007) FAD mutants unable to increase neurotoxic Abeta 42 suggest that mutation effects on neurodegeneration may be independent of effects on Abeta. J Neurochem 101:674-681.

Song W, Nadeau P, Yuan M, Yang X, Shen J, Yankner BA (1999) Proteolytic release and nuclear translocation of Notch-1 are induced by presenilin-1 and impaired by pathogenic presenilin-1 mutations. Proc Natl Acad Sci U S A 96:6959-6963.

Spilman P, Podlutskaya N, Hart MJ, Debnath J, Gorostiza O, Bredesen D, Richardson A, Strong R, Galvan V (2010) Inhibition of mTOR by rapamycin abolishes cognitive deficits and reduces amyloid-beta levels in a mouse model of Alzheimer's disease. PLoS One 5:e9979.

Stiegler P, De Luca A, Bagella L, Giordano A (1998) The COOH-terminal region of $\mathrm{pRb} 2 / \mathrm{p} 130$ binds to histone deacetylase 1 (HDAC1), enhancing transcriptional repression of the E2F-dependent cyclin A promoter. Cancer Res 58:5049-5052.

Sun B, Zhou Y, Halabisky B, Lo I, Cho SH, Mueller-Steiner S, Devidze N, Wang X, Grubb A, Gan L (2008) Cystatin C-cathepsin B axis regulates amyloid beta levels and associated neuronal deficits in an animal model of Alzheimer's disease. Neuron 60:247-257.

Suzuki K, Terry RD (1967) Fine structural localization of acid phosphatase in senile plaques in Alzheimer's presenile dementia. Acta Neuropathol 8:276-284.

Tabuchi K, Chen G, Südhof TC, Shen J (2009) Conditional forebrain inactivation of nicastrin causes progressive memory impairment and agerelated neurodegeneration. J Neurosci 29:7290-7301.

Tu H, Nelson O, Bezprozvanny A, Wang Z, Lee SF, Hao YH, Serneels L, De Strooper B, Yu G, Bezprozvanny I (2006) Presenilins form ER Ca2+ leak channels, a function disrupted by familial Alzheimer's disease-linked mutations. Cell 126:981-993.

Vogt DL, Thomas D, Galvan V, Bredesen DE, Lamb BT, Pimplikar SW (2009) Abnormal neuronal networks and seizure susceptibility in mice overexpressing the APP intracellular domain. Neurobiol Aging. Advance online publication. Retrieved Nov. 1, 2010. doi:10.1016/j.neurobiolaging.2009.09.002.

Wang J, Liu S, Fu Y, Wang JH, Lu Y (2003) Cdk5 activation induces hippocampal CA1 cell death by directly phosphorylating NMDA receptors. Nat Neurosci 6:1039-1047.

Watanabe H, Smith MJ, Heilig E, Beglopoulos V, Kelleher RJ 3rd, Shen J (2009) Indirect regulation of presenilins in CREB-mediated transcription. J Biol Chem 284:13705-13713.

Wen Y, Yu WH, Maloney B, Bailey J, Ma J, Marié I, Maurin T, Wang L, Figueroa H, Herman M, Krishnamurthy P, Liu L, Planel E, Lau LF, Lahiri DK, Duff K (2008) Transcriptional regulation of beta-secretase by p25/ cdk5 leads to enhanced amyloidogenic processing. Neuron 57:680-690.

Wiley JC, Hudson M, Kanning KC, Schecterson LC, Bothwell M (2005) Fa- 
milial Alzheimer's disease mutations inhibit gamma-secretase-mediated liberation of beta-amyloid precursor protein carboxy-terminal fragment. J Neurochem 94:1189-1201.

Wines-Samuelson M, Schulte EC, Smith MJ, Aoki C, Liu X, Kelleher RJ 3rd, Shen J (2010) Characterization of age-dependent and progressive cortical neuronal degeneration in presenilin conditional mutant mice. PLoS One 5:e10195.

Wong E, Cuervo AM (2010) Autophagy gone awry in neurodegenerative diseases. Nat Neurosci 13:805-811.

Xu J, Litterst C, Georgakopoulos A, Zaganas I, Robakis NK (2009) Peptide EphB2/CTF2 generated by the gamma-secretase processing of EphB2 receptor promotes tyrosine phosphorylation and cell surface localization of $N$-methyl-D-aspartate receptors. J Biol Chem 284:27220-27228.

Yang DS, Kumar A, Stavrides P, Peterson J, Peterhoff CM, Pawlik M, Levy E, Cataldo AM, Nixon RA (2008) Neuronal apoptosis and autophagy crosstalk in aging PS/APP mice, a model of Alzheimer's disease. Am J Pathol 173:665-681.
Yu H, Saura CA, Choi SY, Sun LD, Yang X, Handler M, Kawarabayashi T, Younkin L, Fedeles B, Wilson MA, Younkin S, Kandel ER, Kirkwood A, Shen J (2001) APP processing and synaptic plasticity in presenilin-1 conditional knockout mice. Neuron 31:713-726.

Yu WH, Cuervo AM, Kumar A, Peterhoff CM, Schmidt SD, Lee JH, Mohan PS, Mercken M, Farmery MR, Tjernberg LO, Jiang Y, Duff K, Uchiyama Y, Näslund J, Mathews PM, Cataldo AM, Nixon RA (2005) Macroautophagy — a novel Beta-amyloid peptide-generating pathway activated in Alzheimer's disease. J Cell Biol 171:87-98.

Zhang C, Wu B, Beglopoulos V, Wines-Samuelson M, Zhang D, Dragatsis I, Südhof TC, Shen J (2009) Presenilins are essential for regulating neurotransmitter release. Nature 460:632-636.

Zhang D, Zhang C, Ho A, Kirkwood A, Sudhof TC, Shen J (2010) Inactivation of presenilins causes presynaptic impairment prior to postsynaptic dysfunction. J Neurochem. Advance online publication. Retrieved Nov. 1, 2010. doi:10.1111/j.1471-4159.2010.07011.x. 